ten Gradienten, wenn kein vorheriger Austausch stattgefunden hatte.

Die vorliegende Untersuchung läßt die experimentellen Schwierigkeiten erkennen, die bei der Bestimmung der Proteinsynthese in intakten Pflanzenzellen bestehen. Da die Proteinsynthese sehr schnell verläuft, müßten zur Feststellung des Aktivitätsgradienten sehr kurze Versuchszeiten gewählt werden. Auch bei Verwendung höchst markierter Aminosäuren würden dann nur geringe Aktivitäten in der Peptidkette erscheinen, die unterhalb der Meß- genauigkeit lägen. Ungünstig wirkt sich weiterhin aus, daß sich von den angebotenen Aminosäuren höchstens $30 \%$ im Virusprotein wiederfinden und $\mathrm{da} ß$ der Austausch der freien Aminosäuren nur langsam erfolgt. Bei der Untersuchung zellfreier Systeme werden diese Fehlerquellen wesentlich geringer, doch blieb dann fraglich, inwieweit die in vitro gewonnenen Ergebnisse auf die lebende Zelle übertragbar sind.

Die Arbeit wurde vom US Public Health Service (Grant Nr. 5 R01 CA 04601-07) unterstützt.

\title{
Elektronenanlagerungs-Massenspektrographie und Thion-Thiol-Umlagerung
}

\author{
R. MAYer und P. Rosmus \\ Institut für Organische Chemie der Technischen Universität Dresden \\ M. von Ardenne, K. Steinfelder und R. Tümmler \\ Forschungsinstitut Manfred von Ardenne, Dresden-Weißer-Hirsch \\ (Z. Naturforschg. 22 b, 1291-1295 [1967] ; eingegangen am 13. April 1967)
}

\begin{abstract}
Die Elektronenanlagerungs-Massenspektrographie erwies sich als ein geeignetes Mittel zur Untersuchung der Thion-Thiol-Umlagerung in Thioncarbonaten und Thioharnstoffen. Elementarer Schwefel $\left(S_{8}\right)$ bildet unter den angeführten experimentellen Bedingungen negative Ionen von $S_{2} \ominus$ bis $\mathrm{S}_{6}{ }^{\ominus}$, während $\mathrm{S}_{7} \ominus$. und $\mathrm{S}_{8} \ominus$.Ionen nur in sehr geringer relativer Häufigkeit nachweisbar sind.
\end{abstract}

In der vorliegenden Arbeit wird die Elektronenanlagerungs-Massenspektrographie (EA-Massenspektrographie) ${ }^{1-3}$ zur Untersuchung der Thion-ThiolUmlagerung

$$
-x-c-i d x
$$

von Thioncarbonaten (II) und Thioharnstoffen (III) bzw. (IV) angewendet. Zum Vergleich werden die EA-Massenspektren des Diphenyltrithiocarbonats (I) und des elementaren Schwefels aufgenommen.

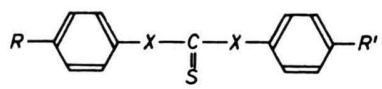

$$
\begin{array}{ll} 
& \mathrm{I}: \quad \mathrm{X}=\mathrm{S} \\
& \mathrm{II}: \quad \mathrm{X}=\mathrm{O} \\
& \mathrm{III}: \quad \mathrm{X}=\mathrm{NH} \\
\mathrm{a}: & \mathrm{R}=\mathrm{R}^{\prime}=\mathrm{H} \\
\mathrm{b}: & \mathrm{R}=\mathrm{R}^{\prime}=\mathrm{CH}_{3} \\
\text { c: } & \mathrm{R}=\mathrm{R}^{\prime}=\mathrm{NO}_{2} \\
\mathrm{~d}: & \mathrm{R}=\mathrm{NO}_{2} ; \quad \mathrm{R}^{\prime}=\mathrm{H} \\
\mathrm{e}: & \mathrm{R}=\mathrm{CH}_{3} \mathrm{O} ; \quad \mathrm{R}^{\prime}=\mathrm{H} \\
\mathrm{f}: & \mathrm{R}=\mathrm{Cl} ; \mathrm{R}^{\prime}=\mathrm{H}
\end{array}
$$

1 M. v. Ardenne, Tab. zur Angew. Phys. Bd. I, Dtsch. Verlag d. Wiss., Berlin 1962.

2 R. Tümmler, Z. physik. Chem. 229, 58 [1965].

3 M. v. Ardenne, K. Steinfelder u. R. Tümmler, Z. Chem. 5, 287 [1965].

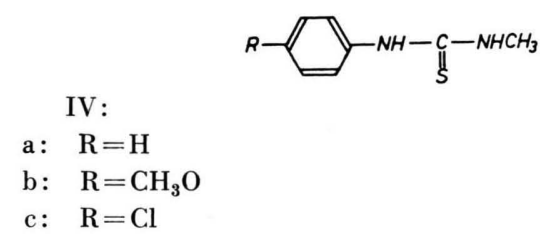

Die hochaufgelösten Spektren der positiven Ionen einiger Vertreter der Typen I und II sind kürzlich von DJerassi et al. ${ }^{4}$ diskutiert worden. Die von uns beobachteten Fragmentierungs-Mechanismen in den EA-Massenspektren der negativen Ionen ähneln denen der durch Elektronenstoß (ES) erzeugten positiven Ionen. EA- und ES-Massenspektren ergänzen sich insofern, als jeweils die schwachen Peaks der einen Ionenart bei der anderen sehr intensiv auftreten. Daraus ergibt sich die Möglichkeit, das charakteristische Verhalten beider Verfahren auch zur Strukturuntersuchung unbekannter Substanzen auszunützen ${ }^{5}$.

4 J. B. Thomson, P. Brown u. C. Djerassi, J. Amer. chem. Soc. 88, 4049 [1966].

5 S. Huneck, C. Djerassi, M. v. Ardenne, K. Steinfelder u. R. Tümmler, Tetrahedron Letters [1967], im Druck. 


\begin{tabular}{|c|c|c|c|c|c|c|c|c|c|}
\hline Nr. & typ & M & $\mathrm{M}-1$ & $\mathrm{PhS}$ & $\mathbf{M}-\mathrm{PhS}$ & $p \mathrm{RPhS}$ & $\mathrm{M}-p \mathrm{RPhS}$ & $p \operatorname{RPhX}^{1} \mathrm{I}$ & M- $p \operatorname{RPh} X$ \\
\hline Ia & & & - & $100(109)$ & & & - & - & . \\
\hline II a & & & $15 \overline{(229)}$ & & & - & - & $100-(93)^{2}$ & $45 \overline{(137)^{2}}$ \\
\hline & & & & & & & - & & $40(13$ \\
\hline II b & $p-\mathrm{CH}_{3} \mathrm{PhOCS}$ & $9 \underset{-}{2}$ & - & - & - & $68(123)$ & - & $100(107)$ & $68(151)$ \\
\hline II c & $p-\mathrm{O}_{2} \mathrm{NPhOCSOPhNO}_{2}-p$ & - & - & $16(109)$ & - & $29(154)$ & - & $100(138)$ & $16(182)$ \\
\hline IId & $p-\mathrm{O}_{2} \mathrm{NPhOCSOPh}$ & $\begin{array}{l}- \\
-\end{array}$ & $\begin{array}{l}- \\
-\end{array}$ & & $2 \overline{(166)}$ & $61(154)$ & $3 \overline{(121)}$ & $100(138)$ & $12(137)$ \\
\hline III a & PhNHCSNHPh & - & $58(227)$ & $21(109)$ & & $\begin{array}{l}- \\
-\end{array}$ & - & $27(92)^{2}$ & $4(136)^{2}$ \\
\hline IIIe & $p$ - $\mathrm{CH}_{3} \mathrm{OPhNHCSNHPh}$ & - & $19(257)$ & $40(109)$ & $-(14$ & $4(139)$ & $\begin{array}{r}58(119) \\
100(118)\end{array}$ & $58(122)$ & $8(136)$ \\
\hline IIIf & $p$-ClPhNHCSNHPh & $52 \overline{(262)}$ & $\begin{array}{l}19(\overline{261})^{3}{ }^{3} 4 \\
59(260) ?\end{array}$ & $\overline{-}$ & - & ${ }^{-3}$ & $-^{3}$ & $2(126)$ & $\overline{-}$ \\
\hline IV a & $\mathrm{PhNHCSNHCH}_{3}$ & - & $29(165)$ & $36(109)$ & $\overline{100}$ & - & - & $8(92)^{2}$ & $5 \quad(74)^{2}$ \\
\hline IVb & $p-\mathrm{CH}_{3} \mathrm{OPhNHCSNHCH}{ }_{3}$ & - & $34(195)$ & - & - & $4 \overline{(139)}$ & $-(57)$ & $12 \overline{(122)}$ & $\begin{array}{l}- \\
-\end{array}$ \\
\hline IV e & $p$ - $\mathrm{ClPhNHCSNHCH}_{3}$ & $100 \overline{(200)}$ & 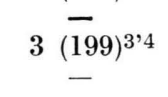 & $\begin{array}{l}- \\
-\end{array}$ & $3 \stackrel{-}{-}(91)$ & $\begin{array}{l}- \\
-3 \\
-\end{array}$ & $\begin{aligned} 100 & (58) \\
- & (57) \\
32 & (58)\end{aligned}$ & $\begin{array}{l}- \\
\overline{-}^{3}\end{array}$ & $\begin{array}{l}- \\
-\end{array}$ \\
\hline
\end{tabular}

Tab. 1. Zusammenstellung der typischen Peaks in den EA-Massenspektren der Substanzen I-IV. Die Intensitäten sind in $\%$ angegeben, bezogen auf den stärksten Peak des Spektrums, zugehörige Massenzahl als Klammerwert. Ein zweiter Wert ist dann angegeben, wenn $\pm 1 \mathrm{ME}$ neben dem zu erwartenden Peak ein anderer mit relativ hoher Intensität auftritt. ${ }^{1} \mathrm{X}=\mathrm{O}, \mathrm{NH}$; ${ }^{2} \mathrm{R}=\mathrm{H} ;{ }^{3}$ bei Cl-substituierten Molekülen treten Schwierigkeiten bei der Deutung der Spektren auf, da das Cl-Atom offensichtlich sehr leicht abgespalten oder an Bruchstücke angelagert werden kann. ${ }^{4}$ Einfluß des Akzeptors auf den Anlagerungsmechanismus.

Die Bildung der negativen Molekülionen durch Anlagerung energiearmer Elektronen erfolgt bei den Verbindungstypen I bis IV vorwiegend nach den beiden Mechanismen ${ }^{6}$

$$
\begin{aligned}
& \mathrm{M}+\mathrm{e} \rightarrow \mathrm{M}^{\ominus}, \\
& \mathrm{M}+\mathrm{e} \rightarrow(\mathrm{M}-1)^{\ominus}+\mathrm{H} .
\end{aligned}
$$

Von den untersuchten Verbindungen sind in Tab. 1 die typischen Peaks der EA-Massenspektren zusammengestellt. Die Intensitätsangaben erfolgen in \%, wobei der stärkste Peak im Spektrum mit 100\% angesetzt wird. Die eingeklammerten Zahlen geben die zugehörige Masse des Ions an. Der in einigen Fällen angegebene zweite Zahlenwert weist auf einen charakteristischen Peak hin, der unmittelbar neben dem zu erwartenden auftritt. Beim Diphenyltrithiocarbonat (I a) ist die relative Tendenz zur Bildung des Molekülions, das hier nach dem RadikalanionMechanismus entsteht, von allen untersuchten Verbindungen am größten. Das steht qualitativ im Einklang mit der zu erwartenden Verschiebung der Elektronen-Affinitäten im Sinne der vereinfachten Vorstellung im HMO-SC Term-Schema ${ }^{7}$, welches spek-

${ }^{6}$ R. Tümmler u. K. Steinfelder, Z. Chem. 7, 1 [1967].

7 M. J. Janssen, Dissertation, T.N.O., Utrecht 1959, J. F Fabian, Dissertation, TU, Dresden 1965.

8 R. Mayer, D. Kunz u. P. Rosmus, in Vorbereitung. troskopisch $^{7}$ und polarographisch ${ }^{8}$ gestützt werden konnte.

In den Abbn. 1, 2 und 3 sind die EA-Massenspektren der drei Grundkörper Diphenyltrithiocarbonat (I), Diphenylthioncarbonat (II) und Diphenylthioharnstoff (III) wiedergegeben. Diese Spektren bestätigen, daß die Thion-Thiol-Umlagerung mit der EA-Massenspektrographie in ähnlicher Weise untersucht werden kann wie mit der ES-Massenspektrometrie $^{4}$. Wie wir fanden, ist die Umlagerung nicht nur bei Thioncarbonaten, sondern auch bei Thioharnstoffen möglich. Die Umlagerung wurde inzwischen auch im EA-Massenspektrum von Thiophenylessigsäuremorpholid $\left(\mathrm{X}=\mathrm{CH}_{2}\right)$ festgestellt. Substituenten am Phenylkern üben im allgemeinen einen charakteristischen Einfluß auf den Spaltungsmechanismus aus. Während man bei Akzeptoren linienarme Spektren erhält, fragmentieren die negativ geladenen Molekülionen mit Donatoren als Substituenten leichter als die nicht substituierten Verbindungen des gleichen Typs.

Wie aus Tab. 1 hervorgeht, erleichtert ein Akzeptor-Substituent in $p$-Stellung des Phenylringes die Umlagerung (II c). Eine unsymmetrische Substitution dirigiert die Umlagerung vorwiegend an die Seite des $p$-ständigen Akzeptors (II d). 


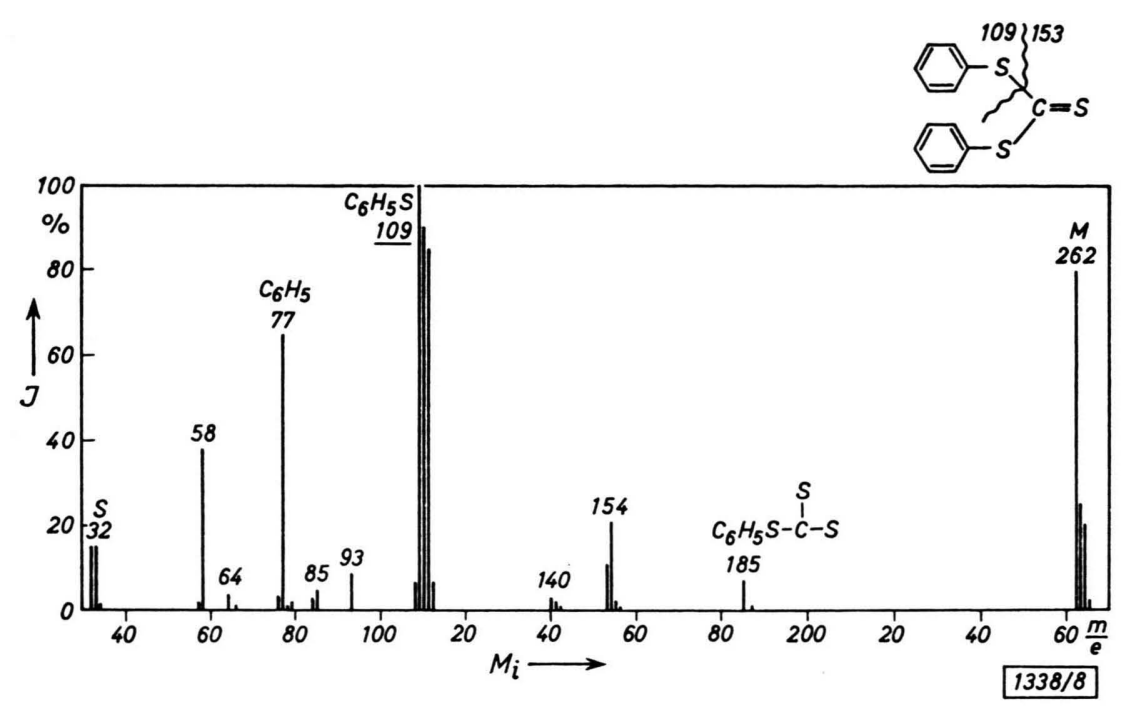

Abb. 1. EA-Massenspektrum des Diphenyltrithiocarbonats (I a) $\mathrm{C}_{13} \mathrm{H}_{10} \mathrm{~S}_{3}$, MG 262; Aufnahmebedingungen: $T_{\mathrm{V}}=20^{\circ} \mathrm{C}, t_{\mathrm{E}}=$ $5 \mathrm{~s}$.

Abb. 2. EA-Massenspektrum des Diphenylthioncarbonats (II a) $\mathrm{C}_{13} \mathrm{H}_{10} \mathrm{O}_{2} \mathrm{~S}$, MG 230; Aufnahmebedingungen: $T_{\mathrm{V}}=20^{\circ} \mathrm{C}, t_{\mathrm{E}}=$ $5 \mathrm{~s}$.
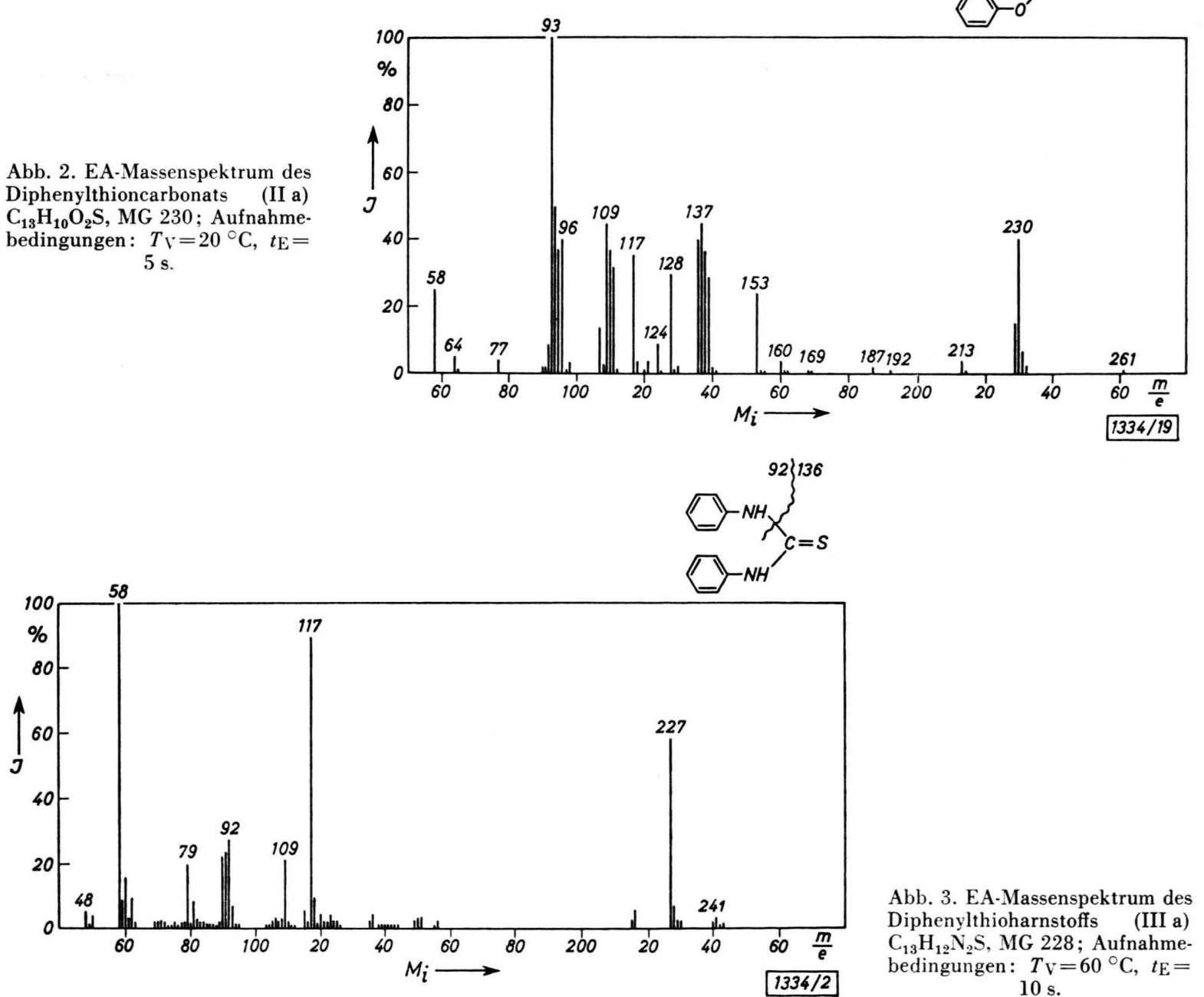

Abb. 3. EA-Massenspektrum des Diphenylthioharnstoffs (III a) $\mathrm{C}_{13} \mathrm{H}_{12} \mathrm{~N}_{2} \mathrm{~S}$, MG 228; Aufnahmebedingungen: $T_{\mathrm{V}}=60^{\circ} \mathrm{C}, t_{\mathrm{E}}=$ $10 \mathrm{~s}$. 
Abb. 4. EA-Massenspektrum des $N$ - 4 - Methoxyphenyl- $N^{\prime}$ - phenylthioharnstoffs (III e)

$\mathrm{C}_{14} \mathrm{H}_{14} \mathrm{~N}_{2} \mathrm{OS}$, MG 258; Aufnahmebedingungen: $T_{\mathrm{V}}=40^{\circ} \mathrm{C}, t_{\mathrm{E}}$

$$
=3 \mathrm{~s} \text {. }
$$

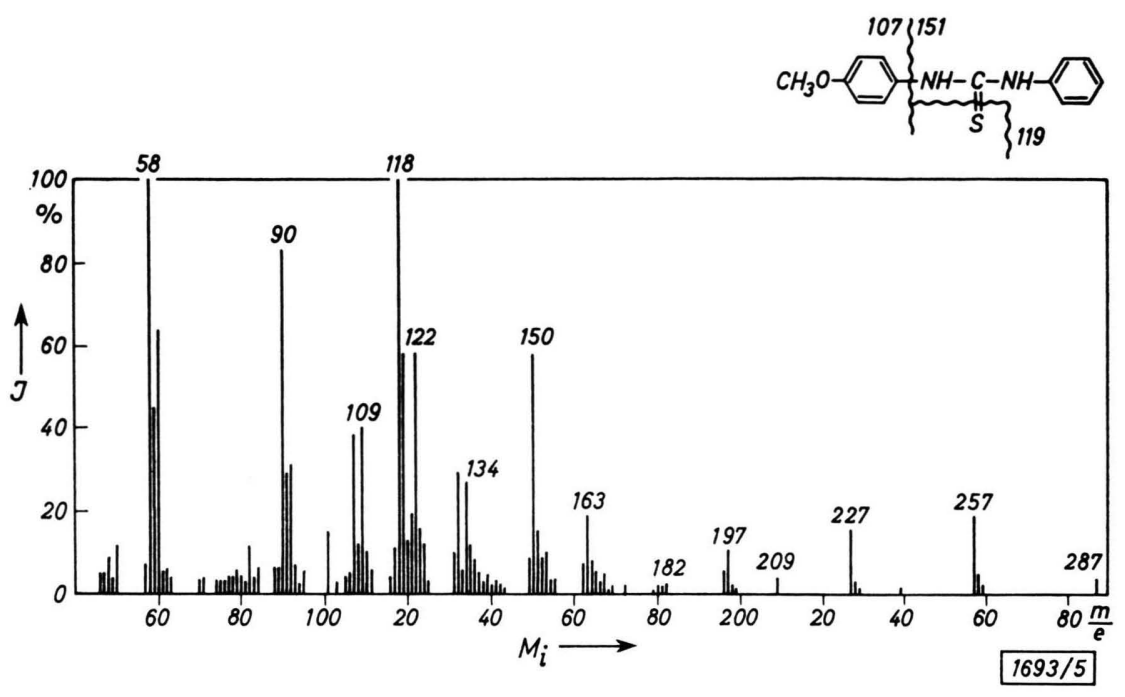

Bei Thioharnstoffen ist der Einfluß der Donatoren größer als bei den Verbindungen des Typs II. Bei der unsymmetrisch substitutierten Verbindung (III e), Abb. 4, entsteht fast ausschließlich ein nichtsubstituiertes Thiophenolat-Ion. Eine Umlagerung an die Seite des Phenylrestes mit $p$-ständigem Donator konnte nur in sehr geringem Umfang beobachtet werden.

An den Chlor-substituierten Verbindungen läßt sich das Verhalten bei der Umlagerung wegen der leichten Beweglichkeit des Chlor-Atoms weniger gut untersuchen.

Der Substituenteneinfluß geht bei der Umlagerung von Diphenylthioncarbonaten (II) konform mit den präparativen Ergebnissen der thermischen ScнöNBERG-Umlagerung in der Gasphase ${ }^{9-13}$. Im Gegensatz zur präparativen Umlagerung haben wir während der gesamten Versuchsdauer bei bedeutend niedrigeren Temperaturen gearbeitet (vgl. l.c. ${ }^{4}$ ). Die Temperatur der Ionenquelle lag bei Zimmertemperatur bzw. infolge Erwärmung während des Betriebes bei $30-40{ }^{\circ} \mathrm{C}$. Zur Verdampfung der Proben sind im allgemeinen Temperaturen von $40^{\circ}$, maximal $100{ }^{\circ} \mathrm{C}$ erforderlich, bei einem Gesamtdruck in der Ionenquelle von $10^{-2}-10^{-3}$ Torr.

Ursachen für das Auftreten der Umlagerung unter den vorliegenden milden Bedingungen könnten

9 A. Schönberg u. L. Vargha, Ber. dtsch. chem. Ges. 63, 178 [1930]; A. Schönberg, L. Vargha u. W. Paul, Liebigs Ann. Chem. 483, 107 [1930].

10 H. K. Al Kazimi u. D. S. Tarbell, J. Amer. chem. Soc. 77, 2479 [1955]. die stoßenergetischen Verhältnisse in der Ionenquelle oder auch eine Erniedrigung der Potentialbarriere im Sinne eines 4-Zentren-Mechanismus sein:

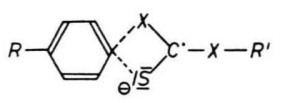

Da bei der Fragmentierung Schwefelbruchstücke auftreten, wurde das Verhalten des elementaren Schwefels $\left(\mathrm{S}_{8}\right)$ bei der Elektronenanlagerung untersucht. Dieselbe Probe von elementarem Schwefel $\left(\mathrm{S}_{8}\right)$ wurde gleichzeitig von uns mit Hilfe der ES-Massenspektrographie untersucht. Es zeigte sich, daß es im Unterschied zur ES-Massenspektrographie bei der Elektronenanlagerung hauptsächlich zur Bildung von $\mathrm{S}_{2}{ }^{\ominus}$ - bis $\mathrm{S}_{6}{ }^{\ominus}$-Bruchstücken kommt. Die Ionen $\mathrm{S}_{7}{ }^{\circ}$ und $\mathrm{S}_{8}{ }^{\ominus}$ treten nur auf sehr intensiven Spektren in Erscheinung und sind etwa um den Faktor 100 schwächer $^{14}$ (Abb. 5). Die Häufigkeit der niedrigeren S-Fragmente nimmt mit steigender Temperatur $\mathrm{zu}$.

\section{Beschreibung der Versuche}

\section{Elektronenanlagerungs-Massenspektrographie}

Zur Aufnahme eines EA-Massenspektrums werden jeweils $0,1-1 \mathrm{mg}$ der Proben in ein einseitig abgeschmolzenes Kapillarröhrchen gefüllt. Dieses Glasröhrchen hat einen Außendurchmesser von $2 \mathrm{~mm}$ und eine

11 D. H. Powers u. D. S. Tarbell, J. Amer. chem. Soc. 78, 70 [1956].

12 H. Kwart u. E. R. Evans, J. org. Chemistry 31, 410 [1966].

13 M. S. Newman, 152nd Meeting Amer. chem. Soc., Sept. 1966, $111 \mathrm{~S}$.

14 I. HERk, Diplomarbeit, TU, Dresden 1967. 


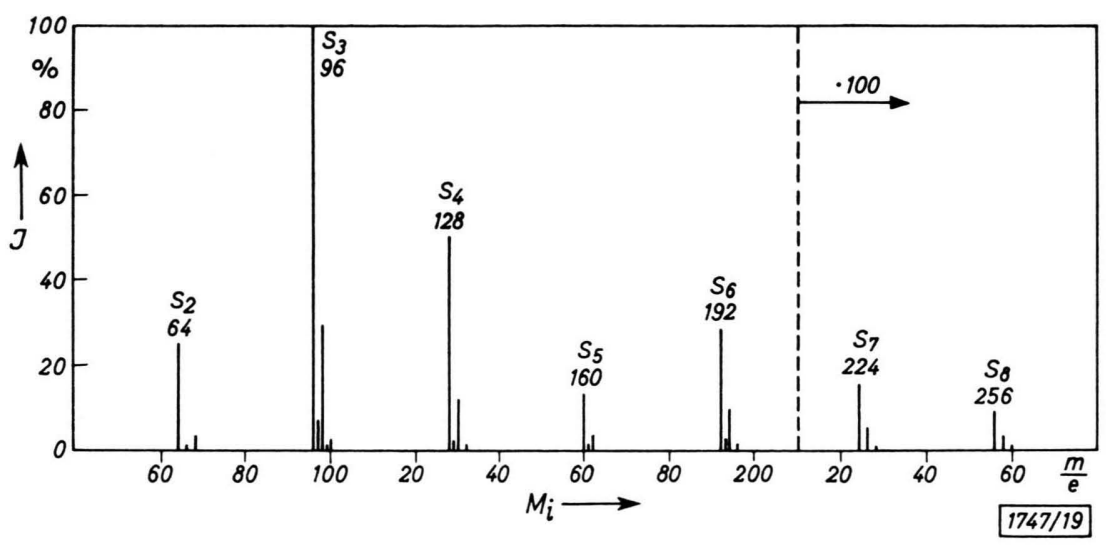

Abb. 5. EA-Massenspektrum des elementaren Schwefels $\mathrm{S}_{8}$; Aufnahmebedingungen: $T_{\mathrm{V}}=40^{\circ} \mathrm{C}$, $t_{\mathrm{E}}=3 \mathrm{~s}$.
Länge von 10-15 mm. Die Verdampfung erfolgt nach dem Einschleusen direkt in der Ionenquelle aus einem heizbaren Verdampfer. Während der Verdampfungsperiode wird in Abhängigkeit von der Verdampfertemperatur $T v$ und der Expositionszeit $t_{\mathrm{E}}$ eine Serie von 10-15 Spektren auf einer Photoplatte registriert. Die abgebildeten EA-Massenspektren stellen Umzeichnungen dar. Úber der Massenzahl $M_{\mathrm{i}}$ ist die jeweilige relative Ionenintensität aufgetragen, wobei die Intensität für den stärksten Peak im Spektrum $\mathrm{J}=100 \%$ gesetzt wird. Einzelne Aufnahmebedingungen s. Text und Bildunterschriften. Vergleiche l. c. ${ }^{\mathbf{1}-\mathbf{3}}$.

\section{Ausgangssubstanzen}

Alle Verbindungen waren bekannt und in Ausbeuten $>70 \%$ leicht darstellbar:

Das Diphenyltrithiocarbonat (I a) und die symmetrisch substituierten Diphenylthioncarbonate (II a, II b, II c) wurden durch Umsetzung des jeweiligen Natrium-thiophenolats bzw. des Natrium-phenolats mit Thiophosgen in Anlehnung an die Literatur 16 hergestellt. Das unsymmetrische $p$-Nitrodiphenylthioncarbonat (II d) entstand durch Reaktion des

15 W. Autenrieth u. H. Hefner, Ber. dtsch. chem. Ges. 58, 2151 [1925].

16 H. Eckenroth u. K. Kock, Ber. dtsch. chem. Ges. 27, 3410 [1894].

17 B. Pawlewski, Ber. dtsch. chem. Ges. 37, 158 [1904].

18 H. King u. I. M. Tonkin, J. chem. Soc. [London] 1946, 1063.
Thiokohlensäure-p-nitrophenylesterchlorids mit Natriumphenolat nach l.c. ${ }^{10}$. Die Thioharnstoffe des Typs III bzw. IV konnten durch Reaktion des Phenyl- bzw. Methylsenföls mit den entsprechenden Anilinen gewonnen werden. Alle Substanzen wurden durch mehrmalige Kristallisation aus Äthanol gereinigt und chromatographisch sowie spektroskopisch auf Reinheit geprüft.

$$
\begin{aligned}
& \text { [Schmp.] [Lit.] } \\
& \text { a: } \mathrm{C}_{13} \mathrm{H}_{10} \mathrm{~S}_{3}(262,4) \quad 96^{\circ} ; \quad 15 \quad 95,5^{\circ} \\
& \text { II a: } \mathrm{C}_{13} \mathrm{H}_{10} \mathrm{O}_{2} \mathrm{~S}(230,3) \quad 106-107^{\circ}{ }^{16} 106^{\circ} \\
& \text { II b : } \mathrm{C}_{15} \mathrm{H}_{14} \mathrm{O}_{2} \mathrm{~S}(258,3) \quad 133^{\circ} ; \quad 16132^{\circ} \\
& \text { II c: } \mathrm{C}_{13} \mathrm{H}_{8} \mathrm{~N}_{2} \mathrm{O}_{6} \mathrm{~S}(320,3) 198^{\circ} ; \quad 10196-197^{\circ} \\
& \text { II d: } \mathrm{C}_{13} \mathrm{H}_{9} \mathrm{NO}_{4} \mathrm{~S}(275,3) 181^{\circ} ; \quad 10181-182^{\circ} \\
& \text { III a: } \mathrm{C}_{13} \mathrm{H}_{12} \mathrm{~N}_{2} \mathrm{~S}(228,3) 154-155^{\circ} ; 17154-155^{\circ} \\
& \text { III e: } \mathrm{C}_{14} \mathrm{H}_{14} \mathrm{~N}_{2} \mathrm{OS}(258,3) 153-154^{\circ} ; 20143^{\circ} \\
& \text { Gef. C 64,75 H 5,56 S 12,31, } \\
& \text { Ber. C } 65,09 \text { H } 5,46 \text { S } 12,41 \text {. } \\
& \text { III f: } \mathrm{C}_{13} \mathrm{H}_{11} \mathrm{CIN}_{2} \mathrm{~S}(262,8) 158^{\circ} ; \quad{ }_{21} 152^{\circ} \\
& \text { Gef. C } 59,26 \text { H } 4,65 \text { S 12,04, } \\
& \text { Ber. C } 59,42 \text { H } 4,22 \text { S } 12,20 \text {. } \\
& \text { IV a: } \mathrm{C}_{8} \mathrm{H}_{10} \mathrm{~N}_{2} \mathrm{~S} \quad(166,3) \quad 114^{\circ} ; \quad{ }_{22} 113^{\circ} \\
& \text { IV b : } \mathrm{C}_{9} \mathrm{H}_{12} \mathrm{~N}_{2} \mathrm{OS}(196,3) 169^{\circ} ; \quad 18169^{\circ} \\
& \text { IV c: } \mathrm{C}_{8} \mathrm{H}_{9} \mathrm{CIN}_{2} \mathrm{~S}(200,7) \quad 145-147^{\circ} ;{ }^{19} 146-147^{\circ}
\end{aligned}
$$

19 R. F. Hunter u. J. W. Jones, J. chem. Soc. [London] 1930, 2190.

20 N. Campbell, B. K. Campbell u. S. J. Patelski, Proc. Indiana Acad. Sci. 53, 119 [1943].

21 C. Kuellin, Ber. dtsch. chem. Ges. 36, 194 [1903].

22 W. Gebhardt, Ber. dtsch. chem. Ges. 17, 3038 [1884]. 